\title{
An Intelligent Robotic Framework for Automated Assembly
}

\author{
H.Y.K. Lau, K.L. Mak and M.C.C. Ngan \\ hyklau@hku.hk, makkl@hku.hk, mccngan@graduate.hku.hk. \\ Department of Industrial and Manufacturing Systems Engineering \\ The University of Hong Kong, Pokfulam Road, Hong Kong
}

\begin{abstract}
Recent applications of robots for industrial automation have shown significant improvement in manufacturing processes in terms of reducing labor participation, enhancing flexibility, efficiency and quality of the products. However, most applications are limited to point-to-point and noninteractive operations in which the availability of a highly structured setup is a prerequisite. This prompts the vast emergency of researches on intelligent robotics that are aimed to improve the adaptability, flexibility and dexterity so as to enhance the intelligence of industrial robots.
\end{abstract}

This paper investigates the designs of intelligent robotic systems and discusses the proposed criteria required to achieve an intelligent robotic system. A proposed conceptual framework for robotic assembly is then presented that contains two main parts, namely, a robotic state recognizer and a control strategy generator. In addition to these two components, the integration of compliant motion control into the framework will be described. An example of using the proposed framework to develop a robotic assembly system is given.

\section{Introduction}

Robotic assembly is regarded as a complicated task. Since the introduction of industrial automation, industrial manipulators have been widely applied to assembly operations. Most robotic operations involve pick-and-place operations that point-to-point motions are merely required. Sophisticated tasks such as peg-in-hole and deburring operations are barriers to high-speed automation. At present, there is no industrial robot that can adapt rapidly to its changing environment and can manipulate objects without a precise definition effectively. Conventionally, control of robots is focused on model-based control such as position and force control. However, the major characteristic that differentiate human and machine is that human are capable of understanding, exploring, reasoning and learning under uncertain environment. In contrast, machine outperforms human under a pre-defined environment. Therefore, some researches have been focused on investigating systems that are capable of transferring and mapping human knowledge to robots [12].

Many methodologies for intelligent control of robots have emerged in the last two decades such as fuzzy logic, neural networks and genetic algorithms. Some of these algorithms have been applied to the control of intelligent robots. However, they can only partially solve the problem. More specifically, the study of intelligent robotics is not only relied on the algorithmic design but it is also related to the issue of system integration that ranges from hardware development, the implementation of control algorithms, and system integration. As such, one of the central aims of this paper is to study the importance of system design and the role it plays in an intelligent robotic system. In particular, this paper focuses on the application of intelligent robot in automated assembly.

In general, a typical robotic assembly consists of three phases of motion, namely, free motion, approach motion and fine motion. Different control techniques are applied to perform different motions. For free motion and approach motion, position-based control is appropriate and adequate. However, the major difficulty in automated assembly is during fine/constrained motions. A fine motion involves contact interactions between the end-effector of a robot and manipulated objects within their environment. During a fine motion, contact force arises. Ideally, each force signal/pattern characterizes a distinguishable contact state. This paper introduces a stochastic model - the hidden Markov model (HMM) - to model contact state with uncertainty $[9,10,11]$. Each well-trained HMM allows a robot to perceive and learn about its environment based on the force signals obtained. Hence, a state recognizer can be built from a number of HMMs to identify contact formation. From an alternative viewpoint, the states identified can provide geometrical information to the robotic controller for understanding its interacting environment. Another main component in the conceptual framework is the control strategy generator (CSG), which is dedicated to infer the control strategy to be used based on the state condition that is sensed by the HMM-based state recognizer. As such, the most appropriate control strategy is selected based on the different contact state.

This paper is structured as follows. A review of intelligent robotic system design is given in Section 2 with their respective characteristics highlighted. In Section 3, a set of criteria for the development of intelligent robotic systems is outlined and a conceptual framework is derived. Three levels, namely, the execution level, control and sensing level, and intelligent level have been incorporated by the framework. An actual implementation of an intelligent 
robotic system for automated assembly, which is developed based on the proposed framework, is given in Section 4 as a case example. The architecture of the robotic system and the hardware setup is also given. Section 5 concludes the paper.

\section{Intelligent Robotic Systems}

Automated robotic systems have been studied for more than two decades. Nevertheless, only slight intelligent robots are commercially available. Difficulties in achieving a truly intelligent robotic system are not only caused by demanding control strategies and complicated robotic work-cells but also lack of formal models for intelligence. From the perspective of system design, there is no unique methodology to model a desirable autonomous system [1]. The suggested models in most literatures are based on either heuristic or analytic approaches. Furthermore, the creditability of autonomous system is unmeasurable. From previous experiences, while developing intelligent robots, concentrating solely on control strategy development is not sufficient. It is also necessary to take into account of system integration. Control strategies should merely be one of the major components in an intelligent system [8]. In essence, system integration is addressed so as to achieve tight integration of hardware and software in an intelligent robotic system domain. In general, system integration is composed of system architecture and system modeling. Three common architectural designs, namely, hierarchical, modular and behavioral design are used. Object-oriented and discrete event methodologies are commonly employed for modeling robotic systems.

\subsection{System Architecture}

A hierarchical intelligent control (HIC) system is proposed in which formal modeling is concentrated on defining and evaluating intelligent systems. Flow of intelligent knowledge (i.e. sensed information either from internal or external sensors) is particularly stressed, instead of knowledge representation. The study of hierarchical intelligent control is adopted from general system theory with the principle known as "Principle of Increasing Intelligence and Decreasing Precision" [1]. Fundamentally, an intelligent robotic system developed in the HIC architecture consists of three levels: the execution level, coordination level and organization level.

For modular design, modular robotics systems are used to model robot resources as distinct functional modules. Such modules are configured selectively into a robot system [5]. Often, object-oriented (OO) modeling is associated with modular robotic systems. Modular design and objectoriented modeling both share many common features such as modularity, flexibility, extensibility and reusability [5].

In a behavioral system, in which robotic motions such as movement and grasping actions, have been modeled $a$ prior, has been developed for more than twenty years [4]. A one-to-one action to behavior mapping characterizes the feature of behavior that is designated to a robot. The behavioral response to the external environment is similar to the action and reaction response of humans. However, this limits the performance of a robot, as each alternation will be reacted by only one action. The intelligence or performance of a robot is thus degraded by this knowledgebased action/reaction mapping system. Besides, the changes in the environment model will require remodel of the characteristic skills of the new behavior based on such changes [9].

\subsection{System Modeling}

Another issue concerning system integration is the adapted modeling technique. The approach to robotic modeling concerns the system modeling of functional modules in accordance to the software aspects. At present, objectoriented models and discrete-event system models are widely used.

\begin{abstract}
Alagar and Periyasamy [13] developed a formal method/specification to model the robotic components such as kinematics, dynamics, sensory system, etc. By using an object-oriented approach, control algorithm can be reused as needed. As an object-oriented approach is used, the reusability is enhanced and is beneficial to the modifiability of such systems. To achieve the desirable level of intelligence, the complexity of the control software and system modeling is highly demanded. One solution to tackle this problem is to use an object-oriented approach together with formal methods to model robotic system with a view to eliminate error in the control software [14].
\end{abstract}

Traditionally, the control algorithms used in most industrial robotic controllers are based on model-based control algorithms in the continuous-time domain. On the other hand, there is another form of control system - the discrete event systems (DES) [9]. Hovland [7] conducted a research of employing DES for selecting a robotic process monitor, which is responsible for identifying assembly states. Taking the merits from both the traditional control and discrete event systems, Huber and Grupen [9] have tried to combine these two controllers into one to form a hybrid discrete event system. They call it the hybrid discrete event dynamic system (DEDS). The hybrid DEDS is partitioned into two parts: the event generator (EG) and the supervisory controller (SC). The event generator analyzes the sensory information arising from physical sensors so as to determine the state of the robot. The state output, which is called as the state space is input to the supervisory controller. The SC is responsible for coordinating mechanical behaviors of a robot for a given task. 


\section{A Conceptual Framework for Intelligent Robotic Systems}

\subsection{Criteria and Characteristics}

Several key elements are commonly found in the literature of intelligent robotic systems (IRS) $[1,4,9,11]$. An ideal IRS is capable of expressing perception, understanding, reasoning and learning. To achieve intelligent control for robots, the following characteristics of intelligent systems are considered:

1. It should be adaptable to deal with unstructured or uncertain environments. An accurate environment model is not required a prior.

2. It should respond to external stimuli in real-time.

3. Error recovery capability should be provided to minimize deviation of actual paths and reduce participation of re-programming robots.

4. The system should provide an emulator that can simulate virtual environment and results of robotic programming.

5. Effective interface is particular important in space exploration and tele-robotics.

6. It should have correct robot models. Fast computation of (inverse) kinematics and (inverse) dynamics are prerequisites for intelligent control. In addition to modeling, precise hardware, robotic sensors are generally required as the foundation of intelligent robot. Correct models and efficient computation can facilitate a robot to respond to external stimuli while performing an operation in real-time.

\subsection{A Proposed Framework}

Since no well-established unified IRS standard has been developed, the design of intelligent systems is mostly taskspecific [10]. Nevertheless, hierarchically structured systems are commonly used. Such systems enjoy consistent information flow as information is sent to low-level modules from high-level modules [1]. A conceptual framework is outlined in Figure 1. In addition, three design principles, namely, (1) robot hardware and robot modeling, (2) control algorithm and sensing, and (3) system integration, are introduced to the framework to address the particular issues arising from each level.

\section{Principle 1-Robot Hardware and Robot Model}

The considerations of robot hardware and robot model are layered in the fundamental level in the proposed framework. On one hand, hardware design and development is the first step in the construction of a robot. Robotic hardware such as actuators, motors and joints determines the accuracy of tasks accomplished by a robot. Precise hardware components enhance the accuracy of control. On the other hand, in order to execute desired behaviours, robot models such as kinematics and dynamics modeling are required for the control of a robot. In order to enhance the efficiency in computing robot models and data processing such as sensed information acquired by industrial sensors, different computer architectures for controlling a robot are extensively researched [4]. These issues are considered in the fundamental development of an intelligent robot.

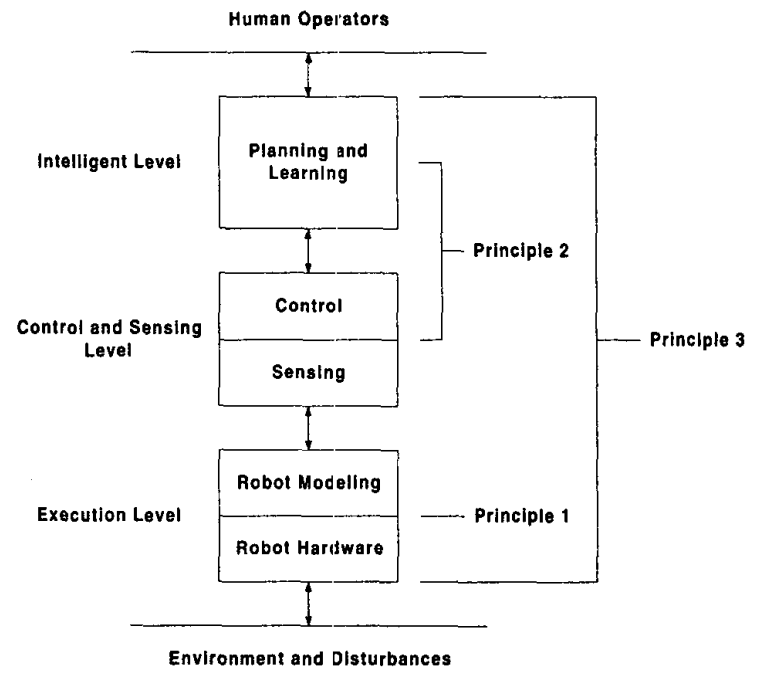

Figure 1: A conceptual framework for IRS.

Principle 2 - Control Algorithms and Sensing Techniques A robot task can basically be divided into two categories, namely, non-contact and contact. The non-contact scenarios are generally performed with position-controlled. However, the contact scenarios complicate the control issue. An accurate robot model and environment model, which describes spatial relationship between a manipulated object and the environment, are not easily acquired. Yet, it is possible to employ suitable sensing techniques to relax such constraints. As such, a well-developed feedback control system is required. With regard to the entire robotic system, control algorithms integrated with sensory information form a closed-loop system, which is used to monitor the accurate accomplishments of robotic tasks. In general, three approaches, namely, explicit mathematical theory (model-based), probability theory and artificial intelligence techniques, are deployed in robotic control.

Model-based control algorithms are related to the mathematical modeling of relationships between actual and sensed information. Hybrid position/force control and compliance control are instances of model-based control algorithms. To attain better intelligence of robots, another approach - the use of artificial intelligence (AI) techniques, is deployed in the last two decades. In retrospect, AI considers the representation of knowledge and reasoning. Recently, algorithmic developments such as neural networks (NNs), genetic algorithms (GAs) and fuzzy logic are categorized in the topic of AI [3]. In contrast to AI, probability-based control involves the uses of probability and statistical theories to model a dynamic environment, rather than using symbolic expressions. At present, this class of control has attracted much interest [4]. A special 
class of stochastic models that is originally deployed in speech recognition called hidden Markov models (HMMs) is used in the proposed research to continuously recognize spatial state information during robotic assembly. This is the basis for the state recognizer (SR) that provides the capability of perceiving the assembly states of a robot undergoing an assembly operation.

\section{Principle 3 - System Integration}

System architecture and system modeling constitute the study of system integration. These two issues are closely related to system implementation. Architecture denotes the characterization and structure of robotic systems. Modeling concerns the representation of modules designed in the system with respect to hardware and software design domain.

\section{An Implementation of an IRS with an Industrial Robot}

The conceptual framework for developing intelligent robotic systems is outlined in Section 3. In order to demonstrate the heterogeneity of the framework, the design and implementation of a practical intelligent automated assembly system is undertaken. There are two intelligent components that are focused in the proposed system (Figure 2). The first intelligent component is the HMM-based state recognizer (HMM-SR) and the second is the rule-based control strategy controller (CSG). Their functions are discussed in the following sections. The system in Figure 2 is structured in a hierarchical manner and the design is based on the conceptual framework depicted in Figure 1.

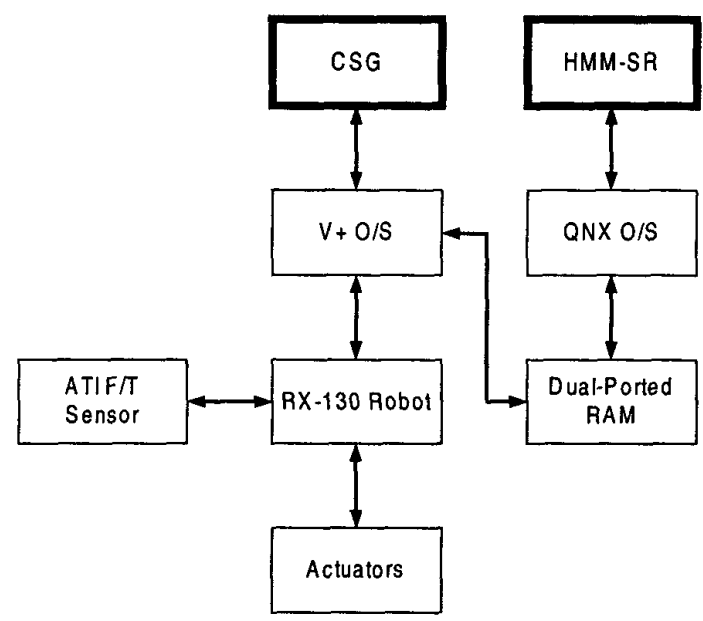

Figure 2: Hardware and software components of the proposed IRS.

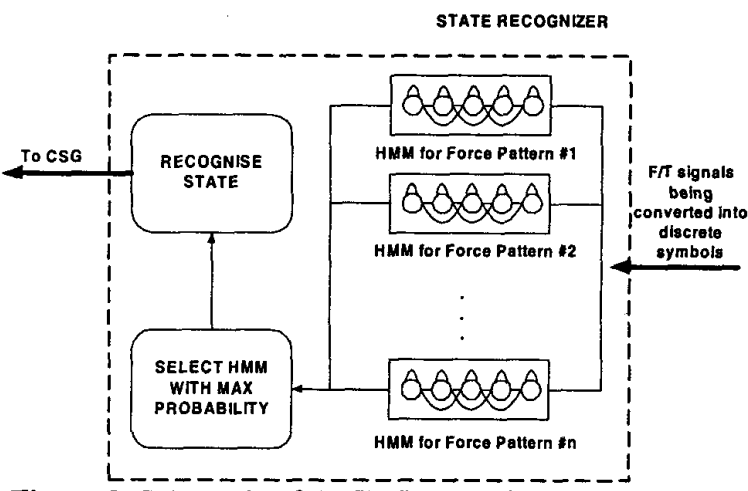

Figure 3: Schematic of the HMM-based state recognizer.

\subsection{HMM-based State Recognizer}

As an intelligent agent, the proposed HMM-based robotic controller has the ability to sense and recognize its current state of operations [8]. Moreover, this controller also has the capability to lean and reason. Learning is an adaptive process in which unmodeled factors can be learnt from gained experience. Reasoning is referred to the capability to understand vague information from unstructured environment. In robotic assembly, a pure positioncontrolled robot is not suitable to perform assembly operations that often involve non-contact and contact formations. As assembly process requires the interactions between the environments and other objects, it is reasonable to use a force-controlled robot. The measured force/torque signals can be used to model the contact scenarios as the formulation of these signals emulates another form of position information.

An HMM is chosen as the statistical model to describe a state formation during an assembly process. A distinct HMM is used to represent a particular state formation. By building a set of HMMs for all the representative assembly states that reflect the spatial information of assembly, a state recognizer can be constructed. By matching the unknown states with the representative states, the state recognizer can therefore identify the unknown state. As a result, the recognized states generated from an HMM-based state recognizer can help to make an accurate identification of assembly states for the motion controller or the control strategy generator, which is described in the next section. The mechanism of selecting a state with an HMM-based state recognizer is shown in Figure 3. The output of the state-recognizer is spatial position information between contacting objects.

\subsection{Control Strategy Generator (CSG)}

An assembly operation consists of three phases, namely, gross motion, approach motion and fine motion. Different control strategies can be used in these three phases. The objective of the CSG is to generate a reference motion strategy to the robot that is based on a particular input state generated from the state recognizer. Alternatively, CSG is 
responsible for motion planning, which facilitates the selections of gross motion and fine motion control schemes based on the current state information. While an endeffector holding a peg is far away from a hole, it is recommended to use gross motion control strategies. On the other hand, if a peg makes a contact with a hole, it is recommended to use fine motion control such as force control. The main advantage of this approach to command generation is to shorten assembly time. It is also advantageous that the impact forces are minimized and the possibility of damaging objects can be avoided as force strategy is selected appropriately and transition from position control to force control is made smoothly.

When comparing with other approaches to assembly operation planning [15], the proposed CSG simply involves a look-up table. Criteria of selecting the most appropriate control strategy are based on the selection of the highest confidence level in the look-up table. The look-up table can be regarded as a rule-based/knowledge-based system that stores mappings between contact states and corresponding control strategies. Note that each contact state identified from the HMM-based state recognizer has its own look-up table and the confidence level is determined by the prior knowledge of the engineers/designers. Table 1 shows an example of a representation of a constrained contact state.

Table 1: Representation of constrained contact state.

\begin{tabular}{|l|c|}
\hline Control Strategies (Label) & Confidence Level \\
\hline Position (S1) & 0.2 \\
Pure Force (S2) & 0.4 \\
Stiffness (S3) & 0.6 \\
Impedance (S4) & 0.8 \\
Hybrid (S5) & 1.0 \\
\hline
\end{tabular}

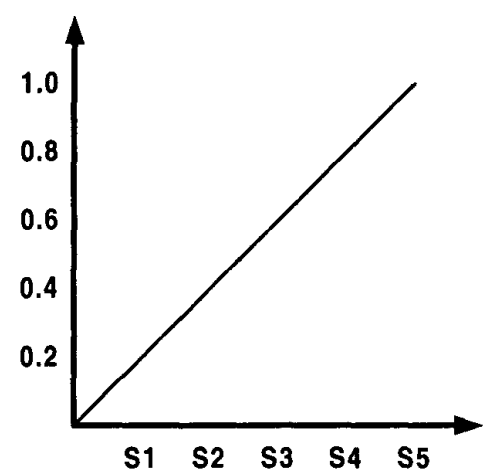

Figure 4: Diagrammatic representation of Table 1.

The use of a simple look-up table is beneficial to fast data transfer between the HMM-based state recognizer and the CSG. In addition, engineering knowledge can be injected into the look-up table. This means that human knowledge can be mapped easily to a robot control system. One possible future development of the CSG is to extend the current system to deploy a fuzzy inference system that can infer the confidence level in the fuzzy set domain.

\subsection{System Architecture}

The developed robotic system for intelligent automated assembly is shown in Figure 4. The design is based on the general architecture for intelligent robotic systems that is outlined in Figures 1 and 2.

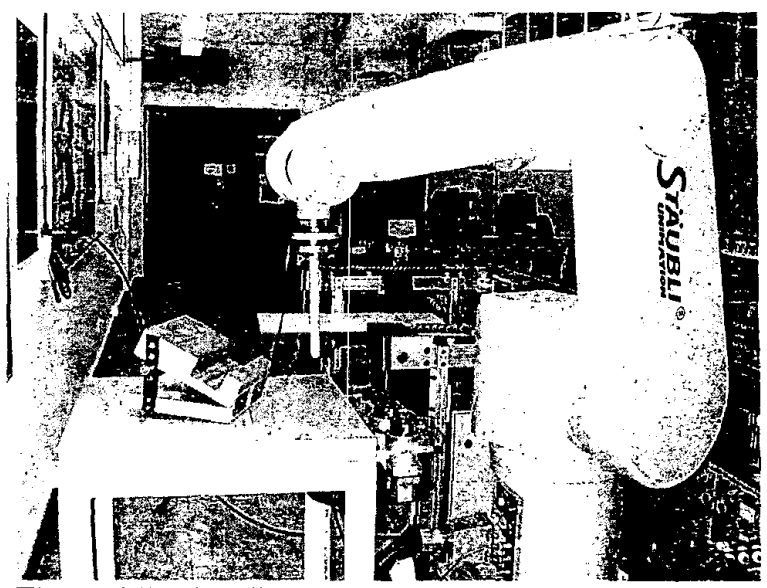

Figure 4: The intelligent robotic assembly system based on a Staubli RX130 industrial robot.

The architecture of the assembly system is structured into three levels, namely, the execution level, the control and sensing level, and the intelligent level. A Staubli RX130 industrial robot is deployed as the assembly robot. The controller hardware is modified according to Figure 2. A Xycom XVME-653, Intel Pentium PC board is integrated to the Adept MV controller so as to provide a suitable platform for the implementation of the hierarchical control framework, and to enhance the computation throughput. Figure 6 shows the hardw/are arrangement of the modified control system, with the PC board shown highlighted.

Base on this hardware architecture, the execution level of control is implemented by the original Adept-based controller under the $\mathrm{V}+$ operating system. This level of control is responsible for joint motion and trajectory control. At the control and sensing level where the HMMbased state recognizer is situated, is implemented on the Pentium PC board. The flexibility of the Pentium PC board together with the QNX real-time operating system facilitates the implementation. The information shared between the Adept controller and the Pentium PC is efficiently and effectively transferred over the dual-ported RAM that resides on the PC board (Figure 7).

Finally, the control strategy generator (CSG) is attributed to the intelligent level. A database of high-level control algorithms including position, force, and hybrid control algorithms is implemented. The CSG is implemented to infer the most appropriate control strategies based on the state information identified by the state recognizer. The CSG is also implemented on the PC board with the software coded in $\mathrm{C} / \mathrm{C}++$ for portability and efficiency. 


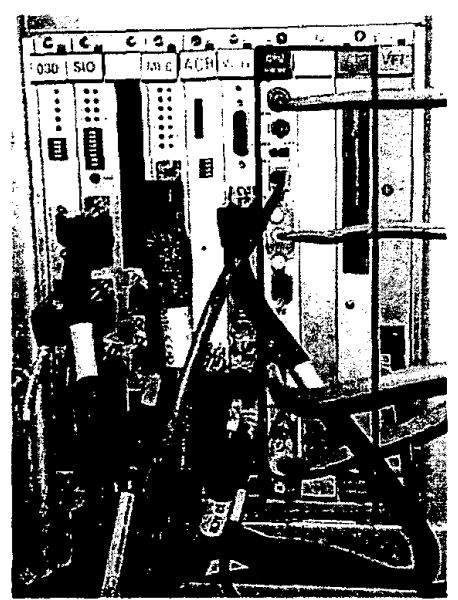

Figure 6:The modified robot controller.

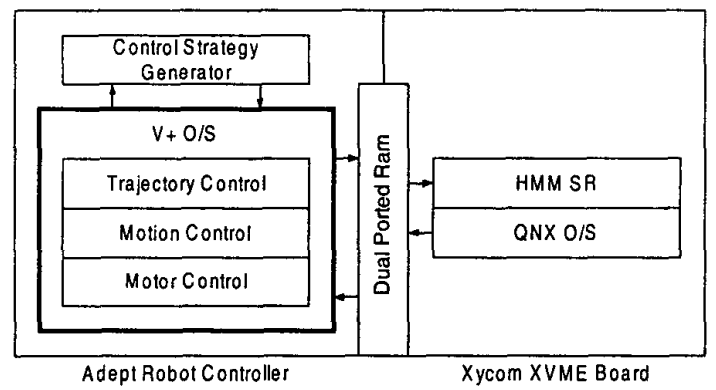

Figure 7: Software architecture of the modified industrial robot controller for the IRS.

\section{Conclusions}

This paper investigates the essences of intelligent robotic systems. A hierarchically structured framework for developing IRS is proposed. The design issues are solved using three essential principles. A generic intelligent robotic system is introduced and characteristics of such the system are discussed. Two major components of such system are outlined, namely, the HMM-based state recognizer and control strategy generator. The state recognizer is responsible for sensing assembly states of the operations whereas the strategy generator is designed for inferring the most appropriate choices of control strategies. An implementation of a practical intelligent assembly system based on an industrial robot is discussed and a full implementation and assembly trials are currently being undertaken.

\section{Acknowledgements}

This research is funded by the Hong Kong Research Grant Council (RGC) under the grant HKU 7075/98E.

\section{Reference}

[1] Valavanis, K. P. and Saridis, G. N., Intelligent robotic systems: theory, decision and applications, Kluwer
Academic Publishers (1991).

[2] Hannaford, B. and Lee, P., 'Hidden Markov model analysis of force/torque information in telemanipulation'. International Journal of Robotics Research, Vol. 10, No. 5 (1991), pp. 528 - 539.

[3] Harris, C. J., Moore, C. G. and Brown, M., Intelligent control: aspects of fuzzy logic and neural nets, World Scientific (1993).

[4] Brady, M., Gerhardt, L. A. and Davidson, H. F., Robotics and Artificial Intelligence, Springer-Verlag (1984).

[5] Fryer, J. A., McKee, G. T. and Schenker, P. S., 'Configuring robots from modules: an object oriented approach'. Proceedings of the 1997 IEEE International Conference on Robotics and Automation, pp. $907-912$.

[6] Brooks, R. A. 'A robust layered control system for a mobile robot', A. I. Memo 864, Artificial Intelligence Laboratory, Massachusetts Institute of Technology, (1985).

[7] Hovland, G. E., Control of sensory perception for discrete event systems, Ph.D. Thesis, The Australian National University, Australia (1997).

[8] Lau, H. Y. K. Mak, K. L. and Ngan, M. C. C., 'A hierarchical HMM-based framework for intelligent robotic assembly'. Proceedings of the 4th Annual International Conference on Industrial Engineering Theory, Applications and Practice (1999), San Antonio, Texas, USA.

[9] Huber, M. and Grupen, R. A., 'A hybrid discrete event dynamic systems approach to robot control'. Technical Report No. 96-43, Laboratory for Perceptual Robotics, Department of Computer Science, University of Massachusetts (1996).

[10] Albus, J. S., 'Outline for a theory of intelligence'. IEEE Transactions on Systems, Man, and Cybernetics, Vol. 21, No. 3 (November, 1991), pp. 473 - 509.

[11] Janabi-Sharift, F. and Wilson, W. J., 'An intelligent assembly robotic system based on relative pose measurements'. Journal of Intelligent and Robotic Systems, Vol. 12 (1996), pp. 49 - 86.

[12] Kiguchi, K. and Fukuda, T., 'Intelligent position/force controller for industrial robot manipulators applications of fuzzy neural networks'. IEEE Transactions on Industrial Electronics, Vol. 44 No. 6 (December, 1997), pp. 753 - 761.

[13] Achuthan, R., Alagar, V. S. and Radhakrishnan, T., 'An object-oriented modeling of real-time robotic assembly system'. First IEEE International Conference on Engineering of Complex Computer Systems (1995), pp. $310-313$.

[14]Alagar, V. S. and Periyasamy, K., 'Formal specifications are mathematical models: an example from robotics'. Proceedings of the 1992 IEEE/RSJ International Conference on Intelligent Robots and Systems, Raleigh, N. C. (July, 1992), pp. 733 - 741.

[15] Bagchi, S., Planning under uncertainty by spreading activation through an adaptive probabilistic network. Ph.D. Thesis, Center for Intelligent Systems, Vanderbilt University, USA (1994). 